\title{
Les réformes curriculaires à l'épreuve de la réalité: du pilotage à la gouvernance
}

\section{Michel Carbonneau}

Au-delà de la question de leur pertinence, les réformes curriculaires présentent l'inexorable défi de leur mise en ouvre, défi qui se mesure à l'aune de leur envergure. Le sujet est ici examiné à partir d'une étude de cas, celle de l'implantation d'une vaste et profonde réforme des programmes scolaires du primaire et du secondaire entreprise au Québec au début du nouveau millénaire. Il est analysé sous l'angle de la nature et de l'ampleur des actions de pilotage que nécessite une telle opération. Onze constats et extrapolations en découlent qui constituent autant de leçons dégagées de cette expérience. Ils réfêrent aux acteurs du milieu scolaire, aux mesures particulières à mettre en place et à l'exercice de pilotage proprement dit. Leur présentation est enrichie de références à diverses études connexes.

\section{Introduction}

La mise en œuvre des réformes curriculaires peut bien se nourrir d'optimisme, elle se déroule rarement comme le souhaiteraient les politiques et emprunte peu souvent les voies imaginées par les stratèges. Néanmoins, d'une expérience à l'autre, on tente de tirer leçon des essais et erreurs précédents afin de maximiser les chances que l'implantation de nouveaux programmes et de nouvelles approches se fasse avec le moins de distorsions possible et dans les temps les plus courts. Celle engagée au Québec est encore trop récente pour qu'il soit possible d'en tirer des leçons définitives, appuyées sur des enquêtes systématiques. Toutefois, le suivi dont elle a été l'objet, assimilable à une étude de cas, permet d'en éclairer le déploiement. À partir de l'examen de situations concrètes, le texte qui suit s'attache à dégager des éléments susceptibles de contribuer à la compréhension des enjeux sous-jacents. 


\section{La réforme curriculaire au Québec ${ }^{1}$}

La réforme curriculaire actuellement en chantier constitue la plus importante réforme des programmes scolaires du primaire et du secondaire à survenir en trente ans au Québec. Tous les programmes, sans exception, ont été l'objet d'une réécriture. On est passé de programmes par objectifs à des programmes par compétences, de programmes disciplinaires cloisonnés à des programmes réunis dans un tout plus homogène, d'un paradigme de l'enseignement à un paradigme de l'apprentissage et enfin, de cycles annuels à des cycles de deux ans.

Le Ministère de l'Education assure la maîtrise d'œuvre de cette vaste opération et il s'est imposé, pour sa réalisation, deux grandes contraintes, celle de respecter un calendrier serré et celle d'implanter, cycle après cycle, à la limite année après année, les nouveaux programmes simultanément dans toutes les écoles. Le processus est à peine amorcé au secondaire, si bien qu'il y a encore peu à dire sur cet ordre d'enseignement. À l'opposé, la situation au primaire, dont l'origine remonte à 1997 , permet de jeter un regard rétrospectif déjà riche en informations et en leçons.

De l'automne 1997 à l'automne 2003, six années se sont écoulées qui ont été le théâtre d'actions nombreuses aux résultats inégaux. Conception et écriture, étalées sur quatre ans, ont donné lieu à d'incessants jeux d'ajustements et réajustements tandis qu'appropriation et implantation ont connu maints tiraillements, en particulier le boycott par les syndicats d'enseignants dont l'objectif premier était d'exercer une pression sur le Ministère en période de négociation de convention collective, mais qui n'en a pas moins affecté le processus de réforme.

À l'origine, les responsables du dossier se sont engagés dans l'aventure convaincus de la nécessité d'opérer un virage dans les programmes scolaires, notamment pour contrer un important taux de décrochage au secondaire, et persuadés du bien-fondé des visées et orientations retenues pour les nouveaux programmes. Si la détermination était au rendez-vous, toutes les conditions n'étaient cependant pas réunies pour assurer la réussite de l'entreprise. De multiples mesures ont dû être mises en place au fil des ans pour y parvenir, encore que tout ne soit pas joué puisque des changements d'une telle ampleur mettent une décennie et plus à s'installer.

\section{L'implantation de la réforme en rétrospective}

Trois grandes étapes ont marqué l'implantation de cette réforme curriculaire. Celle des actions centralisées du Ministère, celle des actions concertées et celle des délégations contrôlées.

\section{Actions centralisées du Ministère: automne 1997 - printemps 2000}

La première étape a été caractérisée par le tâtonnement inhérent à toute action d'une pareille envergure dont on a voulu anticiper l'évolution mais dont la complexité s'est avérée plus grande que prévu. La logistique initiale ne prenait pas en compte un nombre suffisant d'éléments pour être pleinement efficace. 
Pourtant, 19 écoles, dites ciblées, mettaient à l'essai les premières versions des nouveaux programmes, plus de 400 enseignants avaient contribué à les créer soit à titre de concepteurs-rédacteurs, soit à titre de consultants, et de vastes rencontres à portée nationale étaient périodiquement tenues auprès d'administrateurs de commissions scolaires, de conseillers pédagogiques et d'enseignants pour les informer et susciter leur engagement. Concrètement, toutefois, l'équipe responsable du dossier ne comptait pas suffisamment d'effectifs, si bien qu'elle ne parvenait pas à anticiper sur les événements. Trop souvent placée en position de réaction, elle n'arrivait que difficilement à garder le contrôle de la situation et à imposer son plan d'action. Et la difficulté s'est trouvée amplifiée par le fait que l'opération était largement menée «d'en haut», le Ministère initiant la grande majorité des actions engagées dans le cadre de la réforme.

Que s'est-il passé dans les faits? Alors que la réforme était attendue de tous elle était l'aboutissement d'une vaste consultation sous forme d'États généraux sur l'éducation (Ministère de l'Education, 1996) - les critiques ont fusé de toutes parts et aucun aspect n'a été épargné.

\section{En résumé}

1 Les initiatives du Ministère étaient pertinentes, mais disjointes et insuffisantes. Un plan d'action intégré faisait défaut qui aurait permis de mieux les agencer et d'ajouter à l'éventail.

2 Une cellule ou instance centrale de pilotage était manquante. La haute direction du Ministère n'avait pas les ressources requises pour planifier, lancer et suivre les multiples actions que nécessitait le changement entrepris.

3 La gestion de l'implantation de la réforme était trop centralisée, ne laissant pas suffisamment voix au chapitre aux commissions scolaires, voire aux écoles.

\section{Actions concertées: automne 2000 - printemps 2002}

La deuxième étape a été l'occasion pour le Ministère de rajuster le tir. De nouveaux personnels ont été ajoutés à la haute direction, et diverses structures ont été créées dont un comité de soutien à la rédaction des programmes, une table ${ }^{2}$ nationale de concertation et de suivi avec le milieu, un comité central de soutien à l'implantation de la réforme, une table d'instrumentation à l'adresse d'intervenants des commissions et une table d'orientation réunissant des gens du Ministère et les directions des commissions scolaires.

Les objectifs visés étaient nombreux et touchaient, entre autres, la planification et la coordination des actions, la diffusion de l'information, le soutien aux commissions scolaires, le développement d'outils et de stratégies en aide à l'implantation et l'harmonisation des actions associées à la réforme avec les différents autres projets et interventions du Ministère.

Les mesures adoptées ont eu pour conséquence la production de programmes de meilleure qualité (Carbonneau, 2001; 2003), une couverture médiatique plus 
favorable, des réactions syndicales plus pondérées et moins revendicatrices, et une manifestation formelle et publique d'appui à la réforme, notamment de la part des directions d'écoles. Les commissions scolaires se sont par ailleurs impliquées davantage dans l'implantation de la réforme.

\section{En résumé}

1 Un pilotage plus serré a permis de reprendre le contrôle de la situation.

2 La place faite aux partenaires du milieu scolaire a contribué à l'instauration d'un climat de collaboration.

3 La multiplication des actions a créé un mouvement en faveur de la réforme.

4 Une plus grande concertation a grandement amélioré la situation sans répondre totalement aux besoins des commissions scolaires jalouses de leurs pouvoirs et désireuses de jouer un rôle plus important encore.

\section{Délégations contrôlées: automne 2002 - printemps 2004}

La troisième étape a marqué une nouvelle transition dans la conduite de l'implantation de la réforme qui a atteint en quelque sorte sa vitesse de croisière. Les premières années ayant été celles de tous les débats et, d'une certaine manière, de tous les combats, cette nouvelle étape s'est plutôt inscrite dans la continuité et la consolidation. Le défi n'était plus tant d'assurer la survie du projet que d'en garantir la qualité et l'enracinement. À compter de cette période, non seulement la pertinence, mais également l'orientation choisie pour la réforme curriculaire n'étaient plus remises en question, si ce n'est par quelques îlots de résistance n'exerçant plus une grande influence.

Deux grandes préoccupations demeuraient néanmoins, soit faire en sorte que l'implantation amorcée au primaire se poursuive et même s'intensifie et que s'engage correctement celle du secondaire. Sans négliger pour autant le primaire, le Ministère a concentré davantage les ressources et les énergies sur le secondaire. Il en est résulté une prise en charge accrue par les commissions scolaires de l'implantation au primaire, supportées dans leur démarche par les Directions régionales du Ministère sans toutefois leur être inféodées.

Par ailleurs, l'attention portée au secondaire a donné lieu à de nouveaux partages de responsabilités entre Ministère et commissions scolaires. Bien que conservant l'essentiel du pilotage de la réforme, le Ministère a davantage délégué. Une démarche systématique de validation de la version provisoire des programmes a également été menée qui a fait appel à l'expertise d'universitaires aussi bien que de gens du milieu scolaire. Enfin, une table nationale de pilotage a été mise sur pied, composée d'administrateurs du Ministère et de commissions scolaires.

Pour l'avenir, le Ministère projette d'offrir des formations pour chacune des disciplines. L'initiative résulte du constat d'une relative insuffisance des formations offertes à ce jour et constitue, en raison de son origine ministérielle, l'exception dans la mouvance décentralisatrice. 


\section{En résumé}

1 La collaboration des commissions scolaires s'est grandement accentuée à partir du moment où elles ont été davantage impliquées dans la gestion de composantes du plan d'ensemble.

2 Maintenir le tempo de l'implantation de la réforme au primaire tout en amorçant la réforme au secondaire ne s'en est pas moins avéré difficile.

3 Délégation et partage de responsabilités ont entraîné une clarification des rôles des acteurs institutionnels qui a été favorable au changement.

\section{Les actions marquantes}

Au cours de ces six années, quatre initiatives ont été garantes de la poursuite jusqu'à ce jour de l'implantation de la réforme: la création de comités élargis de conception de programmes, la sélection d'écoles ciblées, l'organisation de rencontres nationales et la tenue de séances de validation des programmes de premier cycle du secondaire. Elles ont en commun d'avoir sollicité la collaboration d'un nombre élevé d'acteurs de différents niveaux et de différentes provenances qui, ayant pu influencer le parcours des choses, ont pris fait et cause pour la réforme amorçant ainsi un mouvement pro-réforme qui a eu raison des oppositions.

\section{Constats et extrapolations}

Cette rétrospective ne présente d'intérêt que dans la mesure où on peut en dégager quelques grands enseignements. Nous avons tenté de le faire en formulant des constats et des extrapolations donnant lieu à la formulation de onze leçons regroupées sous trois rubriques. La première réfere aux acteurs du milieu scolaire, la seconde, aux mesures particulières qui s'imposent lors de la mise en œuvre d'une réforme et la troisième, au pilotage, pièce maîtresse d'une telle mise en œuvre.

\section{À propos des acteurs}

\section{Une formation continue et un accompagnement en guise de réforme}

Il est illusoire de penser qu'une fois l'information diffusée et l'adhésion obtenue, la transformation des pratiques suivra spontanément. Rien n'est moins évident et ce, d'autant moins que les changements de paradigmes projetés sont importants (Gauthier \& Saint-Jacques, 2002). Dans le cas de la vaste majorité des enseignants, un support leur sera nécessaire pour s'approprier l'esprit de la réforme et apprendre à le traduire en actions concrètes.

L'implantation de la réforme curriculaire québécoise a été l'occasion de constater que le support à offrir ne pouvait être défini d'en haut. Dans la logique de cette réforme, chaque enseignant et chaque milieu doit construire une com- 
préhension individuelle et collective du nouveau curriculum (Conseil Supérieur de l'Education [CSE], 2003), ce qui ne peut se faire que de manière décentralisée et différenciée.

«L'accompagnement est la réforme» (Rioux-Dolan, 2002). Paraphrasant McLuhan, la formule peut paraître lapidaire, mais elle rend bien compte de la réalité. Elle a été utilisée par la Directrice du service responsable des programmes scolaires pour rappeler que tout projet de réforme curriculaire sera dans les faits à l'image même de ce que les enseignants en auront compris. En ce sens, formation continue et accompagnement contribuent tout autant que le curriculum officiel à déterminer le changement dans sa nature et son contenu non seulement parce qu'ils en véhiculent la teneur mais aussi parce qu'ils constituent en soi des modèles susceptibles d'inspirer les actions futures des personnes en formation.

\section{Leçon:}

La formation et l'accompagnement constituent des éléments clés de la mise en œuvre de réformes curriculaires. Ils doivent donc épouser, aussi bien dans la forme que sur le fond, les visées et orientations du programme-curriculum, seule façon d'assurer un minimum de correspondance entre le projet et la réalité.

\section{La valorisation du jugement professionnel}

Le débat n'est pas clos et pourrait durer longtemps. L'intérêt, cependant, ne tient pas tant à son improbable conclusion qu'à l'évolution que l'on observe dans le recours à l'expression programme ou à l'expression curriculum dans différents pays. Aux États-Unis et dans le Royaume-Uni, où elle a d'abord été utilisée, l'expression curriculum référait à l'orientation que l'on voulait donner à l'éducation des jeunes. Bien qu'on lui ait prêté une grande diversité de sens et une portée très variable au fil du temps et selon les auteurs, elle était tout autant affaire de finalités, de buts ou d'objectifs éducatifs - et de moyens de les atteindre -, que de contenu notionnel et de connaissances disciplinaires à faire acquérir (Eisner, 2003; Grégoire, 1999; Hayes Jacobs \& Perkins-Gough, 2003; Leclercq, 1994; Ornstein \& Hunkins, 1993). Quant au terme programme, utilisé dans les pays latins, il était, à l'origine, davantage restreint dans l'étendue de son acception et renvoyait plus directement à l'enseignement.

Au cours des deux à trois dernières décennies, un rapprochement des deux expressions s'est opéré. La notion de programme a progressivement couvert un champ plus large tandis que le curriculum pouvait, dans certains cas, devenir très spécifique. Ce rapprochement s'explique en partie par le fait que le terme curriculum est utilisé par un nombre grandissant de pays et témoigne d'un accord de plus en plus grand sur l'idée qu'un système d'éducation doit d'un côté assurer l'acquisition de savoirs et d'un autre, favoriser le développement d'habiletés et compétences multiples qui transcendent les disciplines.

Dans la mesure où les objectifs dévolus à l'école et aux enseignants débordent le cadre restreint, pour ne pas dire technique, d'une liste fermée de connaissances 
à maîtriser et où, pour en favoriser l'atteinte, les enseignants doivent jouer les rôles de maître, de mentor, de guide et d'accompagnateur sur des aspects aussi divers que le développement cognitif, affectif, social et citoyen (Goodlad, 2003), ni le contenu d'un curriculum ni son appropriation par les enseignants ne sauraient se limiter à la maîtrise d'habiletés dûment sériées et cataloguées (Carbonneau, 1993; Carbonneau \& Hétu, 1998).

La voie du jugement professionnel, fondé sur des compétences professionnelles encore insuffisamment et inégalement développées, s'avère incontournable pour répondre à cette nouvelle exigence. Les enseignants devront en effet juger de la meilleure façon de faire, compte tenu de leur contexte scolaire propre, pour hisser au niveau de compétences larges et transférables des apprentissages notionnels qui, si on en croit la tendance, continueront d'être, notamment déployés en standards, objet de contrôles de la part des autorités gouvernementales.

\section{Leçon:}

S’il ne convient pas d'emprunter les voies réductrices avec les élèves, à plus forte raison faut-il les éviter avec les enseignants. Leur statut dans le système doit en être un de professionnels invités à conduire leur pratique à la lumière de leur jugement plutôt que dans la soumission à des règles tatillonnes. La mise en œuvre d'un curriculum, dans le sens large de terme, devrait être l'occasion pour eux de développer de nouveaux outils intellectuels et non d'appliquer de nouvelles procédures.

\section{Un espace temporel à créer}

Un temps pour s'approprier, un temps pour approfondir, un temps pour implanter, un temps pour réagir, un temps pour réfléchir, un temps pour se concerter... des temps pour souffler ! Vivre un changement dérange, d'un dérangement intérieur et extérieur. Les habitudes établies qui s'étaient avérées efficaces et économiques ne sont plus que partiellement exploitables. L'environnement professionnel se transforme, il faut penser autrement, agir autrement, bref l'économie générale du travail est bouleversée. Le défi est stimulant pour les uns, écrasant pour les autres. Information, formation et accompagnement sont incontournables dans ces périodes de transition, mais ils réduisent l'espace de liberté et s'ajoutent aux bouffe-temps directement associés à l'apprentissage de nouvelles façons de faire. Au total, on a l'impression que le temps fait défaut de cruelle façon.

Il n'est pas nécessaire que tout le travail additionnel requis par l'appropriation et l'implantation de la réforme trouve place à l'intérieur des heures normales de travail. Les enseignants et les directions d'école sont sensibles au fait que les autorités reconnaissent concrètement le surcroît de travail exigé. Ils ne demandent pas que ce surcroît soit compensé dans sa totalité, mais qu'il le soit dans les limites du raisonnable, ce qui pourra prendre des formes variées selon les milieux.

À ce besoin d'un temps personnel, s'ajoute celui d'un temps institutionnel, 
c'est-à-dire d'un calendrier d'implantation qui ne presse pas indûment, qui laisse le temps de respirer. Entendre les doléances à ce chapitre et ralentir le rythme, à l'occasion, aide beaucoup en rassurant les partenaires sur le fait qu'ils peuvent être entendus.

\section{Leçon:}

À trop exiger, on risque de ne rien obtenir. Conscience et engagement professionnels ont leur limite, si bien qu'on ne peut demander aux acteurs terrain de procéder sous pression constante et d'absorber la totalité des coûts en temps additionnel associés à la mise en œuvre d'une réforme.

\section{À propos des mesures particulières}

\section{Le poids de l'évaluation}

Le thème de la conciliation entre l'aide à l'apprentissage et la réponse à des standards nationaux est l'occasion de vifs débats dont on peut douter que les défenseurs d'une pédagogie de la réussite soient en train de sortir vainqueurs (Barber, 2003). Comment croire, cependant, que les enjeux socio-économiques qui se profilent derrière la réflexion sur les curriculum puissent ramener le pendule des finalités de l'école à la simple maîtrise des savoirs notionnels (Eisner, 2003)? Non seulement ce serait nier les acquis des sciences de l'éducation des dernières décennies, mais ce serait faire abstraction de l'accélération du développement des connaissances et de leur complexification.

La logique d'une approche par compétences, qui caractérise à des degrés divers bon nombre des réformes présentement engagées dans le monde occidental, implique la poursuite d'objectifs de développement globaux et le recours à des situations pédagogiques à connotations heuristiques (Carbonneau \& Legendre, 2002). De telles façons de faire s'accommodent mal d'évaluations étroites centrées sur la maîtrise de connaissances disciplinaires rigoureusement hiérarchisées.

Les services d'évaluation ont donc fort à faire pour développer des modes d'évaluation qui mettent en cause des situations complexes et exigent de l'apprenant qu'il use de compétences larges et polyvalentes. Il faut en effet éviter qu'évaluations et contrôles ne sapent à la base les efforts de renouvellement de la pédagogie, que la recherche fait ressortir comme essentiels, pour satisfaire des besoins, tout aussi légitimes, de qualification optimale du capital humain d'une société.

\section{Leçon:}

Le champ de l'évaluation s'avère un élément particulièrement sensible en ce qu'il détermine, dans les faits, le poids relatif qu'auront les visées de développement et les objectifs de performance d'un système sur l'orientation pédagogique des écoles. Il n'est pas facile aux gens du terrain d'influencer le politique, voire l'exécutif. Il faut néanmoins continuer de prêter oreille aux messages émanant de la réalité scolaire et les tenir en contrepoids aux diktats de la réalité économique 
dont l'importance ne doit pas faire oublier les autres dimensions de la vie, éventuellement contributives à son essor. La mise en place d'une réforme ne saurait faire abstraction de ces enjeux, au risque de s'échouer sur les berges de la pensée à courte vue.

\section{Le recours à des stratégies de changement éprouvées}

Il existe une pléthore d'ouvrages sur l'implantation du changement. Ils sont de portée variable, de qualité variable également, aussi incombe-t-il à chaque milieu de trouver les instruments qui lui conviendront le mieux. Ces moyens et techniques reposent sur des fondements très divers: théorie des systèmes, du fonctionnement des institutions, des comportements humains, du changement, de la gestion des entreprises, etc. (Brassard, 2003; Collerette \& Schneider, 1996; Fullan, 2001; Gossen \& Anderson, 1998; Gustin, 2001; Lafortune \& Deaudelin, 2001; Schneider, Collerette \& Legris, 2002). Leur utilisation dans un contexte particulier suppose toujours une transposition, une adaptation à la situation (Sylvestre, 1998). Le recours à de tels outils ne saurait donc dispenser de la réflexion préalable sur leur adéquation avec l'objectif poursuivi (Bouvier, 1998).

Il n'existe pas de panacée en la matière et aucun outil ne pourra se substituer à l'effort d'analyse et de suivi du processus de mise en œuvre d'une réforme. Une théorie de référence, une grille de lecture, une méthode et des stratégies utilisées à bon escient peuvent toutefois rendre de grands services.

\section{Leçon:}

Ici comme ailleurs, il faut subordonner les moyens à la fin, cerner d'abord les visées, puis choisir les moyens d'action appropriés. En la matière, il importe principalement d'éviter de faire des moyens une fin en soi. Ce risque est élevé dans le monde de l'éducation où l'existence d'un idéalisme certain nourrit l'illusion tenace de trouver des solutions magiques.

\section{Des ressources à la clé}

Espaces appropriés, documentation pertinente et matériels didactiques adaptés contribuent pour beaucoup à la mise en œuvre d'une réforme. Non seulement ils ajoutent à l'impression de sérieux, mais ils fournissent les armes dont ont tant besoin ceux qui quotidiennement se retrouvent sur le front de l'implantation.

Dans le cas de la réforme curriculaire du Québec, la question du matériel didactique est celle qui a été l'objet des plus grandes attentes et du plus grand nombre de tractations. Au dire de certains formateurs du Ministère et des commissions scolaires, la période «sans manuel à l'appui» aura été féconde dans la redéfinition d'un matériel didactique assurant un meilleur soutien à la réforme. Cette réforme, qui préconise la responsabilisation de l'apprenant, la différenciation pédagogique et l'ouverture pédagogique s'accommode assez mal, en effet, de la littérature scolaire classique de type «livre unique» tel que le manuel scolaire ou le cahier d'exercice. Une nouvelle génération de ressources didactiques, davan- 
tage en harmonie avec les nouveaux programmes, doit en accompagner l'implantation si on souhaite sa réalisation dans les meilleurs délais.

\section{Leçon:}

Quand l'intendance ne suit pas, il devient difficile d'avancer sur le terrain. Le soutien matériel ne saurait donc totalement le céder au soutien sur le plan des ressources humaines. Le changement de cap qu'impose une réforme engage la conception d'une instrumentation renouvelée, accordée aux nouveaux défis professionnels. Une attention doit dès lors lui être accordée, à la fois pour répondre aux attentes et pour s'assurer de la cohérence de l'un en regard de l'autre.

\section{À propos du pilotage}

\section{La nécessité d'approche systémique}

Lors de l'implantation d'une réforme curriculaire, enseignants et administrateurs concentrent d'abord les efforts sur la compréhension et l'assimilation des nouveaux éléments en présence. On cherche à identifier les changements de pratique qui en découleront et à introduire les nouvelles façons de faire en bouleversant le moins possible l'organisation en place. Or, dans le cas d'une réforme d'envergure comme celle entreprise au Québec, il s'avère que ces efforts ne suffisent pas, fussent-ils supportés par d'importantes actions de formation continue.

Parce que contraints à développer de nouvelles approches, tant les directions d'école que les conseillers pédagogiques et les enseignants voient leurs tâches et leurs conditions de travail se transformer de manière substantielle (Carbonneau, 2004; Carbonneau \& Giroux, 2004). Les services de gestion des ressources humaines doivent en conséquence s'efforcer d'apporter à l'organisation du travail les ajustements qui s'imposent.

Puisque l'école est un bien public, les premiers intéressés, notamment les parents, devraient avoir voix au chapitre aussi bien au moment de la conception que de l'implantation d'une réforme et ce, d'autant plus que celle-ci est d'envergure. Leur adhésion au changement constitue un atout majeur à sa réalisation.

De la même manière, les partenaires, généralement universitaires, responsables de la formation initiale des enseignants auront un apport d'autant plus déterminant qu'ils seront partie prenante de la conception de la réforme et qu'ils seront mis à contribution au moment de son implantation.

\section{Leçon:}

Pour que la mise en œuvre d'une réforme ait les meilleures chances de réussite, il faut l'aborder avec une vision systémique. Ce ne sont pas les seules instances et services pédagogiques qui doivent être mis à contribution, mais tout ce qui gravite autour de l'organisation scolaire, du fonctionnement de l'école et de la prestation enseignante. Et rien ne doit être négligé pour s'associer les partenaires, notamment les associations de parents et les universités. 


\section{L'importance de miser sur l'effet d'entraînement}

L'introduction d'un changement est beaucoup affaire de rythme et d'équilibre à trouver et à conserver. Mettre en œuvre un nouveau curriculum implique plusieurs décisions aux conséquences souvent difficiles à anticiper. Quel niveau d'adhésion faut-il anticiper de la part des enseignants avant de démarrer une réforme? Comment mobiliser la communauté éducative derrière le projet? Est-il préférable d'y aller année après année du cursus scolaire ou viser une implantation plus massive, par cycle ou par ordre d'enseignement? Quel temps réserver à l'appropriation des nouveaux programmes? Quel degré de pénétration des nouvelles approches exiger les premières années? Comment soutenir l'intérêt à l'endroit de la réforme tout au long de son implantation et maintenir la motivation chez ceux qui devront attendre encore quelques années avant que ne vienne leur tour de l'implanter?

Il n'y a pas de réponses toutes prêtes à ces questions. Elles varient selon les systèmes, selon les milieux et selon la nature des changements que l'on veut introduire. Un constat se dégage néanmoins à l'effet qu'il faut une masse critique de personnes qui bougent en même temps. Si la mise en œuvre progressive paraît souhaitable parce qu'elle permet, en principe, de travailler plus en profondeur, elle présente l'inconvénient de donner à penser que les autorités hésitent, qu'on a tout son temps pour voir venir ou qu'une généralisation à l'ensemble du système pourrait ne jamais survenir.

\section{Leçon:}

Parvenir à créer un momentum autour d'un projet de réforme constitue un atout majeur dans le déroulement de sa mise en œuvre. Susciter l'adhésion, c'est travailler sur un terrain qui s'apparente à celui de la mode. $S^{\prime}$ il faut se méfier du caractère trop souvent superficiel de l'engouement à l'endroit des modes, on peut retenir du mécanisme sous-jacent que «l'effet d'entraînement» est un moteur puissant.

\section{Des réseaux qui trament et qui arment}

L'expérience québécoise des écoles ciblées au primaire a eu pour premier principe actif de créer un réseau de directions d'écoles. L'initiative en a été prise par le Ministère de l'Education pour améliorer l'écriture des programmes, mais les retombées les plus importantes ont certainement résidé dans les conséquences de l'existence de ce réseau sur la mobilisation de ses membres en faveur de la réforme.

Des réseaux dans le monde de l'éducation (Organisation de coopération et de développement économiques [OCDE], 2003), il s'en trouve plusieurs, encouragés par la facilité qu'offrent les technologies de l'information et de la communication. Ils sont internationaux ou locaux, de grande ou petite envergure, quasi permanents ou ponctuels, structurés ou spontanés, avec ou sans port d'attache. Leur prolifération rappelle un peu ce que De Rosnay (1995) qualifie d'organisation fractale, en référence à l'émergence spontanée d'actions apparentées plus ou moins convergentes sans toutefois être globalement concertées. 
En raison de leur nombre sans cesse croissant et de leur impact sur les pratiques éducatives, ils sont en passe de devenir une force au sein des systèmes d'éducation. Ils méritent attention car ils contribuent pour beaucoup au dynamisme du milieu tout en constituant parfois une source d'inquiétude pour les pouvoirs exécutifs puisque susceptibles de servir la résistance au changement.

\section{Leçon:}

Il faut savoir que les acteurs du milieu scolaire ne demandent plus qu'on leur dicte ce qu'il faut faire. Professionnalisation et technologie aidant, ils vont prendre toute initiative de nature à améliorer la qualité et l'efficacité de leurs interventions sans attendre les autorisations d'en haut. La prolifération des réseaux en témoigne. Il s'agit là d'un atout additionnel avec lequel les gestionnaires et administrateurs doivent apprendre à jouer.

\section{Un pilotage adaptatif au service de l'implantation}

Réformes sur papier et réformes sur terrain font deux. Fullan (2001) aborde le sujet en tentant de répondre à la question «Why planning fails» (p.95) et d'autres auteurs tels Brassard (2003) et Perrenoud (1998) rappellent comment la mise en œuvre de réformes en éducation est une entreprise périlleuse. Un bon pilotage est un pilotage adaptatif. Malgré toutes les précautions prises, un plan de réforme et sa traduction en projet sur papier demeurent des modèles abstraits. Leur appropriation par les répondants du milieu scolaire y introduit inévitablement du «bruit». Aussi, faut-il éviter le piège de l'orthodoxie et du conformisme à tout prix. Le temps se charge de dégager sur le terrain les aspects porteurs d'une réforme. Non pas qu'il faille renoncer à ses objectifs, mais que l'on doive accepter l'épreuve de la réalité.

Par ailleurs, rien ne vaut de bonnes mesures de suivi sur le terrain et une posture de recherche pour garantir au pilotage une dimension adaptative. Dans le cas du Québec, ces mesures ont pris la forme de mises à l'essai, de validation, de concertation et de recherche-action. D'autres moyens auraient pu être choisis, l'important étant d'assurer un monitoring constant afin de pouvoir rajuster le tir au moment opportun.

\section{Leçon:}

Le mieux est l'ennemi du bien. Dans tout changement impliquant l'intervention de personnes, surtout lorsque leur nombre est élevé, il faut savoir adapter les mesures d'implantation à l'accueil que leur réservent les principaux acteurs. Entre le report d'échéances, le changement de stratégie, la reformulation d'objectifs, l'assouplissement des contrôles, il faut savoir réajuster les plans de pilotage. Une évaluation continue de l'évolution de la situation demeure indispensable pour garder le cap et divers moyens doivent être pris à cette fin, mais l'élément primordial demeure que le plus grand nombre se mette en démarche et en cheminement. Et seul un pilotage "apprenant» rend possible l'atteinte de cet objectif. 


\section{Le pouvoir du pourquoi}

Lieu commun ou trivialité, l'expérience enseigne que l'explicitation du pourquoi d'une réforme demeure le socle du processus d'implantation. Rares sont ceux qui se souviennent, près de 10 ans plus tard, des origines du changement. La référence aux États généraux (Ministère de l'Education, 1996), pourtant à la source de la réforme curriculaire au Québec, évoque peu de souvenirs dans l'esprit de bon nombre d'enseignants.

Dans le feu de l'action s'installe facilement une dynamique de résolution de problèmes, bonne en soi, mais qui centrée sur l'immédiat se met parfois à tourner sur elle-même. Une dérive insensible amène alors à oublier plus ou moins la réforme au profit d'une analyse de situation et d'une quête de solutions qui ont peu à voir avec la démarche initiale. Revenir constamment sur les intentions premières derrière la réforme et derrière chacune des actions entreprises pour son implantation évite les détours inutiles et favorise la convergence et la complémentarité des interventions.

\section{Leçon:}

La satisfaction du succès à court terme, malgré les dérives dont celui-ci est parfois porteur, l'emporte souvent sur la satisfaction que procure la réussite à moyen et à long terme. Aussi, le retour fréquent aux intentions de départ demeure-t-il essentiel au maintien du cap en plus de constituer une bonne façon d'accroître la résistance à la frustration en période difficile.

\section{Conclusion}

\section{Le besoin de gouvernance}

La décision la plus déterminante dans la gestion de l'implantation de la réforme québécoise a été de créer une véritable cellule de pilotage au niveau central. C'est sans doute la retombée la plus significative de la deuxième étape du processus d'implantation. Le caractère centralisé de cette cellule, malgré son ouverture certaine aux besoins des gens du terrain, en a cependant réduit la portée et empêché que certains milieux ne se prennent en charge. La troisième étape marque à ce chapitre une évolution de taille puisque le pilotage est devenu partenarial. Le Ministère s'est en effet associé des dirigeants de commissions scolaires et des représentants d'associations de directions d'école qui conjointement et solidairement assureront le pilotage de la mise en ouvre de la réforme. Cette table devient ainsi, pour l'essentiel, un lieu d'analyse de l'évolution de la situation et de définition d'objectifs à atteindre pour lesquels les acteurs du milieu devront prendre les mesures jugées les plus appropriées. Elle constitue en quelque sorte un lieu de coordination des actions du milieu plus qu'un état-major d'où partent les ordres.

Même si on observe à nouveau une tendance vers les vastes réformes (Fullan, 2001), le souci de leur enracinement dans la pratique des acteurs du milieu de- 
vient un objet de préoccupation, non plus seulement comme objectif à long terme, mais comme critère à respecter au moment même de leur conception, ce qu'illustrent bien l'étude et la typologie de Paquay (2002). Cela ouvre naturellement la voie à des modes d'implantation diversifiés respectueux des particularismes locaux. De façon générale, on constate que le dynamisme des forces en présence dans les systèmes d'éducation s'exprime de plus en plus sous la forme de pôles définissant les extrémités de continuum divers: centralisation vs décentralisation, curriculum imposé vs programmes locaux, évaluation standardisée vs reddition de compte, technicisation vs professionnalisation enseignante. Ces pôles font bien ressortir les tensions qui caractérisent les attentes à l'endroit desdits systèmes. Ils rendent illusoire l'idée même d'un embrigadement enseignant visant une pratique monolithique, uniformisée et contrôlée (Tardif \& Carbonneau, 2002) et confortent l'idée qu'il faille trouver de nouveaux modes administratifs et gestionnaires qui tiennent compte de ces tensions et de leur caractère irréductible.

Une expression est de plus en plus utilisée en référence à ces modes de gestion, que l'on retrouve notamment dans les organisations dites apprenantes, où l'on choisit de miser davantage sur la coopération, la concertation, l'imagination et l'intelligence collective que sur la seule clairvoyance de l'autorité pour assurer efficience et efficacité d'une entreprise ou d'une institution. Il s'agit de la gouvernance (Glatter, Mulford \& Shuttleworth, 2003).

Paquet (2004) présente assez bien le mot et l'idée qu'il recouvre. C'est ...un mot dont l'usage correspond aujourd'hui à des réalités et à des besoins inédits. Dans un monde où les ressources, le pouvoir et l'information-connaissance sont largement distribués entre plusieurs mains, personne ne peut plus imposer autocratiquement sa gouverne. [...] Il faut parler de gouvernance pour connoter la coordination nécessaire [...] de tous ces partenaires obligés si l'on veut atteindre un minimum d'efficacité (p.9).

La gouvernance n'exclut pas que l'on doive continuer de diriger un système, mais elle exige qu'une autre forme de partage de responsabilités ou de délégation de pouvoir émerge au sein de cette direction.

\section{Leçon dernière:}

À constater comment, dans les sociétés occidentales, le pouvoir sur l'éducation est diffus, il est difficile d'imaginer qu'une réforme puisse être imposée d'en haut, sans participation des diverses catégories d'acteurs et d'usagers à la prise de décision concernant sa conception et sa réalisation. Les autorités en place, politiques ou exécutives doivent donc trouver de nouvelles façons de diriger et d'introduire les changements en concordance avec les nouveaux rapports de force qui s'installent dans les sociétés et leurs institutions. L'approche de type gouvernance pourrait en être une. 


\section{Notes}

1 L'auteur tient à remercier Louise Bussière, consultante auprès du Ministère de l'Education du Québec, et Michel Aubé, professeur à l'Université de Sherbrooke, à qui il est redevable d'une lecture attentive et éclairée de son manuscrit.

2 Le mot table est fréquemment utilisé au Québec pour référer à un groupe de travail.

\section{Références}

Barber, M. (2003). Objectifs réalisables et défis stratégiques - Point de vue de l'Angleterre sur la reconceptualisation de l'enseignement public. In Réseaux d'innovation. Vers de nouveaux modèles de gestion des écoles et des systèmes (pp.115-133). Paris: OCDE.

Bouvier, A. (1998). Évaluation ou pilotage au sein des organisations de formation? In G. Pelletier (Ed.), L'évaluation institutionnelle de l'éducation: défi, ouverture et impasse (pp. 4981). Montréal: Éditions de l'AFIDES.

Brassard, A. (2003). La gestion de la transformation: un regard sur l'expérience québécoise de réforme du curriculum. [Communication]. Montréal: Université de Montréal, Colloque du CRIFPE.

Carbonneau, M. (1993). Modèles de formation et professionnalisation de l'enseignement: analyse critique de tendances nord-américaines. Revue des sciences de l'éducation, 19, (1), $33-57$.

Carbonneau, M. (2001). Le curriculum: une mise à l'essai suivie [de la réforme de l'éducation au Québec]. Vie pédagogique, 118, 54-57.

Carbonneau, M. (2003). Un mode d'emploi pour implanter un nouveau programme. [http://www.viepedagogique.gouv.qc.ca/articles/modeemploi.pdf].

Carbonneau, M. (2004). Lévaluation en service des enseignants au Québec: entre peurs et velléités de pouvoir. In L. Paquay (Ed.), L'évaluation des enseignants: Tensions et enjeux (pp. 233-256). Paris: L'Harmattan.

Carbonneau, M. \& Giroux, M. (2004). Réforme curriculaire et gestion des ressources humaines. Essai à partir d'études de cas menées au Québec. In : Laderrière P. (Ed.), La gestion des ressources humaines dans l'enseignement. Où en est l'Europe (pp. 185-232). Paris : L'Harmattan.

Carbonneau, M. \& Hétu, J.-C. (1998). Formation pratique des enseignants et Naissance d'une intelligence professionnelle. In L. Paquay, M. Altet, É. Charlier \& P. Perrenoud (Ed.), Former des enseignants professionnels. Quelles stratégies? Quelles compétences? (pp. 7796). Bruxelles: De Boeck.

Carbonneau, M. \& Legendre, M.-F. (2002). Pistes pour une relecture du Programme de formation et de ses référents conceptuels. Vie pédagogique, 123, 12-17.

Collerette, P. \& Schneider, R. (1996). Le pilotage du changement. Sainte-Foy: Presses de l'Université du Québec.

Conseil Supérieur de l'Education. (2003). L'appropriation locale de la réforme: un défi à la mesure de l'école secondaire. Québec: Ministère de l'Education.

De Rosnay, J. (1995). L’homme symbiotique. Paris: Éditions du Seuil.

Eisner, E.W. (2003). Preparing for today and tomorrow. Educational Leadership, 61, 6-10.

Fullan, M. (2001). The new meaning of educational change (third edition). New York: Teachers college press.

Glatter, R., Mulford, B. \& Shuttleworth, D. (2003). Gouvernance, gestion et direction. In Réseaux d'innovation. Vers de nouveaux modèles de gestion des écoles et des systèmes (pp.65-86). Paris: OCDE.

Gauthier, C. \& St-Jacques, D. (2002). La réforme des programmes scolaires au Québec. Québec: Les Presses de l'Université Laval 
Goodlad, J.I. (2003). Teaching what we hold sacred. Educational Leadership, 61, 18-21.

Gossen, D. \& Anderson, J. (1998). Amorcer le changement. Un nouveau leadership pour une école qualité. (Traduction de Gervais Sirois). Montréal: Chenelière/McGraw-Hill

Grégoire, R. (1999). La nouvelle piste de l'Oregon. In Le renouvellement du curriculum: expériences américaine, suisse et québécoise. Québec: Conseil Supérieur de l'Éducation.

Gustin, A. (2001). Management des établissements scolaires. De l'évaluation institutionnelle à la gestion stratégique. Bruxelles: DeBoeck Université.

Hayes Jacobs, H. \& Perkins-Gough, D. (2003). Creating a timely curriculum. Educational Leadership, 61, 12-17.

Lafortune, L. \& Deaudelin, C. (2001). Accompagnement socioconstructiviste. Pour s'approprier une réforme en éducation. Sainte-Foy: Presses de l'Université du Québec.

Leclercq, J.-M. (1994). Les programmes d'enseignement en France et à l'étranger. In C. Demonque (Ed.), Qu'est-ce qu'un programme d'enseignement? (pp 145-157). Paris: Hachette.

Ministère de l'Education (1996). Rénover notre système d'éducation: dix chantiers prioritaires. [Rapport final de la Commission des États généraux sur l'éducation]. Québec: Ministère de l'Education.

OCDE (2003). Réseaux d'innovation. Vers de nouveaux modèles de gestion des écoles et des systèmes. Paris: OCDE.

Ornstein, A.C. \& Hunkins, F. (1993). Curriculum, foundations, principles and theory. Boston: Allyn and Bacon.

Paquay, L. (2002). "À quoi bon un curriculum de qualité sill ne change pas les pratiques enseignantes!» [Communication]. Lausanne. Congrès de l'ADMEE.

Paquet, G. (2004). Pathologies de gouvernance. Essai de technologie sociale. Montréal: Liber.

Perrenoud, P. (1998). Évaluer les réformes scolaires, est-ce bien raisonnable? In G. Pelletier (Ed.), L'évaluation institutionnelle de l'éducation: défi, ouverture et impasse (pp. 11-47). Montréal: Éditions de l'AFIDES.

Rioux-Dolan, M. (2002). Les enjeux et défis de l'accompagnement des enseignants en exercice dans le contexte de la réforme. [Communication]. Congrès de l'ACFAS, mai 2002. Québec: Ministère de l'Education.

Schneider, R., Collerette, P, \& Legris, P. (2002). La gestion du changement organisationnel. Deuxième partie. Choisir la stratégie de changement. ISO Management Systems, 2 (2), 4553.

Sylvestre, M. (1998). L'évaluation des établissements d'enseignement: un tour d'horizon. In G. Pelletier (Ed.), L'évaluation institutionnelle de l'éducation: défi, ouverture et impasse (pp. 49-81). Montréal: Éditions de l'AFIDES.

Tardif, M. \& Carbonneau, M. (2002). Au-delà des réformes. In M. Carbonneau \& M. Tardif (Ed.), Les réformes en éducation, leurs impacts sur l'école (pp. 163-177). Sherbrooke: Éditions du CRP.

Liebe Isabelle

Mots clés: réforme, curriculum , pilotage

\section{Curriculare Reformen und ihre Implementation: Prozesse zwischen Steuerun und Führung}

\section{Zusammenfassung}

Curriculare Veränderungen verweisen, jenseits ihrer Relevanz, auf die Anforderungen ihrer Implementierung: je grösser die Veränderungen, desto stärker die 
zu bewältigenden und anstehenden Herausforderungen. Die dazu präsentierte Fallstudie analysiert und diskutiert aktuelle curriculare Entwicklungen der Primar- und Sekundarschulen in Quebec unter dem Gesichtspunkt von Steuerungs- und Implementierungsprozessen und formuliert davon ausgehend zentrale Punkte. Diese beziehen sich auf die Akteure im Bildungssystem, auf den Implementierungsprozess und die entsprechenden Massnahmen. Die Diskussionspunkte werden mit diversen Forschungsergebnissen kommentiert und kontrastiert.

Schlagworte: Curriculumsreform, Steuerung, Implementierungsprozess, Fallstudie

\section{Le riforme curricolari alla prova della realtà: dal pilotaggio alla "governance»}

\section{Riassunto}

Indipendentemente dalla loro pertinenza, le riforme curricolari sono confrontate con l'inesorabile sfida della loro implementazione, sfida che si misura sul filo del loro impatto. La questione viene discussa sulla base di un caso, quello di una vasta e profonda riforma dei programmi scolastici della scuola primaria e secondaria nel Québec all'inizio del nuovo millennio. In particolare si analizzano la natura e l'ampiezza delle attività di pilotaggio che necessita una tale riforma. $\mathrm{Ne}$ derivano dieci constatazioni ed estrapolazioni che corrispondono ad altrettante lezioni che si riferiscono agli attori del contesto scolastico, alle misure specifiche $\mathrm{da}$ attuare e al processo di pilotaggio in senso stretto. Il discorso viene arricchito da riferimenti a diversi studi realizzati in questo campo.

Parole chiave: riforma, curricolo, pilotaggio

\section{Implementation of curricular reforms: management and governance}

\section{Summary}

It is typical of curricular reforms and changes that their implementation is a challenge. The greater the change, the higher is this challenge. A case study concerning the recent implementation of a major change in Quebec's primary and secondary schools curricula shows how such transformation can be carried out. Eleven statements are presented. They refer to the protagonists of the school system, the specific efforts that the implementation process requires, and the management of the process. References to relevant studies are given throughout the paper.

Key words: curricular reform, management, implementation, case study 
T h e m a 